\title{
Odours: What measurement for what diagnosis?
}

- or an industrial plant, odour sources may be very different: channelled effluents (chimneys...), manure spreading basins, decanters, fugitive emissions... Thus, an assessment of the considered site is essential before organizing an analysis campaign. Two different ways for odour measurement can be envisaged, so the choice will be related to the objectives and the information requested.

One alternative approach involves sensory analysis which lead to the determination of odour concentration (olfactometry), odour intensity and annoyance. Most of these methods are the subject of several norms (in France, AFNOR norms NF X 43-101 to NF X 43-104).

At emission, this sensorial approach allows to realise the odour statement and then helps to locate the different sources and to organize them into hierarchy. Consequently, this diagnosis may lead to the process improvement to reduce the olfactory pollution (as example, leeks removal). It permits also to verify the agreement with the regulation.

For the receiving environment, the olfactometric measurements permit to perform impact studies involving the neighbouring inhabitants of the plant. Some complementary information concerning the annoyance, the perception frequency and the unpleasant characteristic of odour can also be obtained through population surveys.

Another analytical alternative consists in physico-chemical methods. In this case, it is possible to identify and to quantify the different molecules which make up the odour. This knowledge is particularly relevant to select the most suited treatment and to determine the dimensions of the deodorization unit. The structural identification of the odorant compounds may also informs about their formation mechanisms. This would lead to solve olfactory pollution by taking action directly on the process, without needing any further treatment. This kind of measurement also helps to estimate the toxicity of the identified molecules.

More recently, multi-sensor systems, also called "electronic noses" show interesting potentialities to estimate "fingerprints" of gaseous samples. However, this technique is not yet sensitive enough for odour purpose and the response obtained is not representative of the multidimensionality of the olfactory information.

At the moment, no method can be considered as a reference for odour measurement, and the different analytical approaches are not in competition but are complementary. In the same way, the following articles clearly present the choice of methods and the related diagnosis through relevant industrial applications and also enlighten some particular sampling cases and analytical limits.

As conclusion, the authors are greatly acknowledged for their relevant contributions and for the time they spent to the elaboration of this special issue.

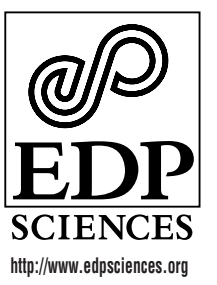

\author{
Coordinated by: V. Desauziers \\ Laboratoire de Génie de l'Environnement \\ École des Mines d'Alès




\section{Odours: What measurement for what diagnosis?}

169 Foreword

V. Desauziers

171 Physicochemical characterisation of odours M. Ramel and M. Nomine

180 Advantages and limits of adsorption sampling for physico-chemical measurements of odorous compounds

J.-M. Guillot, B. Fernandez and P. Le Cloirec

188 Measurement of odours by sensory analysis or "olfactometry" A.-M. Gouronnec and V. Tomasso

199 Laboratory study of odour emissions from areal sources: evaluation of a sampling system C. Leyris, F. Maupetit, J.-M. Guillot, L. Pourtier and J.-L. Fanlo

207 Correspondences between olfactometry, analytical and electronic nose data for 10 indoor paints

0. Ramalho 